\title{
Relation of Urinary Retention and Functional Recovery in Stroke Patients During Rehabilitation Program
}

\author{
Seok Beom Son, MD, Seong Yun Chung, MD, Seok Kang, MD, Joon Shik Yoon, MD, PhD \\ Department of Physical Medicine and Rehabilitation, Korea University Guro Hospital, Seoul, Korea
}

\begin{abstract}
Objective To investigate the relationship between urinary retention and short-term functional recovery in subacute stage after stroke.

Methods The medical records of 94 patients admitted to the rehabilitation unit of Korea University Guro Hospital were reviewed retrospectively. The postvoid residual urine (PVR) was measured at least once a day using a bladder scan, and urinary retention (UR) was defined when the daily PVR volume consistently checked more than $100 \mathrm{~mL}$. Clinical data and functional outcomes of patients in the rehabilitation ward were collected. Functional outcomes were measured using the Mini-Mental State Examination (MMSE), Berg Balance Scale (BBS), Functional Ambulation Category (FAC) level, Fugl-Meyer Assessment (FMA), and Modified Barthel Index (MBI) at admission (or transfer) and discharge. The data of patients with and without urinary retention were compared and analyzed. Results Of the 94 participants, 25 patients were classified to the UR group and 69 were classified to the non-UR group. At the initial stage of rehabilitation, the scores of MMSE, BBS, FAC, MBI were significantly worse in the UR group $(\mathrm{p}<0.05)$. Both groups showed significant improvements of all functional outcomes after rehabilitation $(p<0.05)$. The non-UR group showed more prominent recovery of BBS, FAC, MBI scores $(p<0.05)$.

Conclusion Urinary retention in post-stroke patients is significantly related to the poor functional status at initial stage of rehabilitation, and also to poor recovery after rehabilitation.
\end{abstract}

Keywords Urinary retention, Stroke, Neurogenic urinary bladder

\section{INTRODUCTION}

Stroke patients commonly experience increased postvoid residual (PVR) volume in the first few weeks $[1,2]$ and the incidence of urinary retention (UR) was reported to be about $21 \%$ when assessed 3 weeks after stroke $[3,4]$. Factors that affect the incidence of UR include male gender, older age, prostate hypertrophy, neurologic diseases,

Received June 13, 2016; Accepted September 2, 2016

Corresponding author: Joon Shik Yoon

Department of Physical Medicine and Rehabilitation, Korea University Guro Hospital, 148 Gurodong-ro, Guro-gu, Seoul 08308, Korea. Tel: +82-2-26261500, Fax: +82-2-2626-1513, E-mail: rehab46@korea.ac.kr

ORCID: Seok Beom Son (http://orcid.org/0000-0003-2409-805X); Seong Yun Chung (http://orcid.org/0000-0003-3414-075X); Seok Kang (http://orcid. org/0000-0001-5614-4175); Joon Shik Yoon (http://orcid.org/0000-0002-8985-279X).

(c) This is an open-access article distributed under the terms of the Creative Commons Attribution Non-Commercial License (http://creativecommons.org/ licenses/by-nc/4.0) which permits unrestricted noncommercial use, distribution, and reproduction in any medium, provided the original work is properly cited. Copyright (C) 2017 by Korean Academy of Rehabilitation Medicine 
diabetes, and use of anticholinergic medications [5-8]. Uncontrolled urinary retention leads to renal impairment, urinary incontinence, and urinary tract infection (UTI), all of which are related to the general condition of the patient $[9,10]$.

Bladder dysfunction in stroke patients is related with functional outcomes. In one study, urinary incontinence was reported to be correlated with the presence of aphasia, cognitive impairment, and poor overall functional status [4]. UTI after acute stroke is also associated with poor short-term outcome [11], although there is no definite evidence of the impact of post-stroke infections on neurological deterioration [12,13]. However, few studies have investigated the relation between functional outcome and urinary retention in stroke patients. One study reported that urinary retention is strongly associated with cognitive impairment, aphasia, and poor functional status [14]. Wu and Baguley [15] have also reported that UR was associated with high morbidity and poor clinical outcome in patients admitted for rehabilitation.

Previous studies have evaluated the functional outcomes and PVR volume only at the early stage of rehabilitation. In our knowledge, no study has investigated the relation between consistent UR and functional recovery after stroke rehabilitation. Thus, the aim of the present study was to investigate the relation between UR and short-term functional recovery in the subacute stage, after stroke rehabilitation program.

\section{MATERIALS AND METHODS}

\section{Subjects}

Medical records of patients were retrospectively reviewed, who had been admitted to the rehabilitation unit of a Korea University Guro Hospital in Seoul, Korea, due to newly diagnosed stroke within 3 months, from January 2014 to December 2015. These patients had undergone an intensive stroke rehabilitation program. Inclusion criteria for patients enrolled in this study were as follows: (1) self-voiding during admission in rehabilitation unit, (2) no other orthopedic or neurological problems affecting functional ability, (3) no evidence of UTI at the time of admission or transfer, (4) no history of prostate hypertrophy, and (5) no history of taking medication related to bladder contractile dysfunction, such as anti-cholinergics and tricyclic antidepressants at the time of admission.
UTI was diagnosed if the presence of at least one symptom (fever $>38^{\circ} \mathrm{C}$, urgency, frequency, dysuria, suprapubic tenderness) and the presence of pyuria ( $\geq 10 \mathrm{WBC} /$ $\mathrm{mL}$ on urinalysis) or urine culture with $\geq 10^{5}$ colonies $/ \mathrm{mL}$ [16]. This study was approved by the Institutional Review Board of the Korea University Guro Hospital.

\section{PVR volume measurement and bladder management}

The PVR of all patients was measured within 30 minutes after voiding at least once a day, using the Bladder Scannner BioCon-700 (Mcube technology Co. Ltd., Seoul, Korea). When the PVR measured $<100 \mathrm{~mL}$ three times consecutively, the monitoring was discontinued. For patients with an indwelling urinary catheter at transfer or admission, the PVR volume was measured when they could void volitionally after catheter removal. The UR was defined as the daily PVR volume being consistently $>100 \mathrm{~mL}[14,17]$. Patients who showed UR during admission were classified to the UR group. Intermittent catheterization was performed for bladder emptying, when the PVR measured $>150 \mathrm{~mL}$. Alpha blockers were prescribed for some patients who complained of frequent urination. All the procedure that related to scanning of PVR, were performed by a well-trained physician.

\section{Clinical data}

The demographic data of the patients such as age, sex, height, weight, and body mass index (BMI) were reviewed. In addition, clinical data were collected, including presence of diabetes mellitus, communication disorder, stroke type and location, and mean length of stay in the acute care and rehabilitation wards. The functional outcome of cognitive function, balance, walking ability, motor recovery, and activities of daily living (ADL) were measured using the Mini-Mental State Examination (MMSE), Berg Balance Scale (BBS), Functional Ambulation Category (FAC) level, Fugl-Meyer Assessment (FMA), and Modified Barthel Index (MBI) at admission (or transfer) and discharge. All the data collected were compared and analyzed between the UR and non-UR group.

\section{Statistical analysis}

Statistical analyses were performed using the SPSS software package ver. 22.0 (IBM SPSS, Armonk, NY, USA). For analysis of demographic data between the groups, independent $\mathrm{t}$-test was performed. The clinical data was 
analyzed using the chi-square test. To assess the improvement of functional outcome after rehabilitation, the paired t-test was performed. Generalized lineal model was used to compare improvement of the functional outcomes between the groups in rehabilitation unit. A probability (p) value of $<0.05$ was considered statistically significant.

\section{RESULTS}

The medical records of 177 stroke patients were reviewed, who were admitted to the rehabilitation unit from January 2014 to December 2015. From these, 94 patients ( 56 male, 38 female) were enrolled for this study. Of the 94 patients, 41 were identified to have UR at transfer or on admission. However, 16 patients had recovered their blad- der function as the PVR was below $100 \mathrm{~mL}$ during admission. Therefore, the remaining 25 patients who showed UR until discharge were classified to the UR group, and 69 patients were designated the non-UR group.

The baseline characteristics of both groups are summarized in Table 1. No significant differences were seen in the demographic characteristics between the groups. Clinical data, such as disease duration, diabetes mellitus, aphasia, and stroke type, were not significantly different between both groups, nor was there any significant difference in stroke lesion. Especially in lesions associated with the micturition center (frontal lobe, pons), no significant difference was observed ( $p>0.05$ ). The UR group showed significantly more incidence of urinary frequency $(\mathrm{p}<0.05)$. UTI was significantly more prevalent in the UR group during admission to the rehabilitation unit,

Table 1. Baseline characteristics of study subjects

\begin{tabular}{|c|c|c|c|}
\hline Variable & UR $(n=25)$ & Non-UR $(n=69)$ & p-value \\
\hline Age (yr) & $67.48 \pm 9.6$ & $61.75 \pm 13.3$ & 0.05 \\
\hline Sex & & & 0.54 \\
\hline Male & 11 & 45 & \\
\hline Female & 14 & 24 & \\
\hline Height (cm) & $157.00 \pm 7.02$ & $159.41 \pm 7.41$ & 0.16 \\
\hline Weight (kg) & $57.76 \pm 5.76$ & $60.48 \pm 8.35$ & 0.13 \\
\hline $\operatorname{BMI}\left(\mathrm{kg} / \mathrm{m}^{2}\right)$ & $23.52 \pm 2.85$ & $23.84 \pm 3.22$ & 0.66 \\
\hline Disease duration (day) & $12.3 \pm 5.0$ & $11.3 \pm 4.4$ & 0.33 \\
\hline Days in rehabilitation unit & $21.2 \pm 9.4$ & $20.3 \pm 7.0$ & 0.59 \\
\hline Diabetes mellitus & $8(32.0)$ & $21(30.4)$ & 0.53 \\
\hline Aphasia & $5(20.0)$ & $13(18.8)$ & 0.55 \\
\hline Stroke side & & & 0.40 \\
\hline Right & 11 & 33 & \\
\hline Left & 12 & 32 & \\
\hline Bilateral & 2 & 4 & \\
\hline Stroke lesion & & & 0.27 \\
\hline Supratentorial & 20 & 49 & \\
\hline Infratentorial & 5 & 20 & \\
\hline Stroke type & & & 0.14 \\
\hline Ischemic & 15 & 51 & \\
\hline Hemorrhagic & 10 & 18 & \\
\hline Urinary frequency & $15(60.0)$ & $21(30.4)$ & $<0.05^{*}$ \\
\hline UTI at rehabilitation unit & $18(72.0)$ & $20(29.0)$ & $<0.01^{*}$ \\
\hline Previous UTI history & $7(28.0)$ & $6(8.7)$ & $<0.05^{*}$ \\
\hline
\end{tabular}

Values are presented as mean \pm standard deviation or number (\%).

UR, urinary retention; BMI, body mass index; UTI, urinary tract infection. ${ }^{*} \mathrm{p}<0.05$. 
as compared to the non-UR group $(\mathrm{p}<0.05)$. In addition, the UR group had significantly more UTI histories before transfer or admission to the rehabilitation unit $(\mathrm{p}<0.05)$.

The comparisons of clinical data were also performed between the 16 patients who had recovered their bladder function and 25 patients with continuous UR (Table 2). The mean bladder recovery period of the patients with transient UR was $2.94 \pm 1.88$ days. There were no significant differences in disease duration, stroke types and location of lesion between the patients with transient UR and continuous UR ( $p>0.05$ ). In addition, the use of alpha blocker or cholinergic was not significantly different between the patients $(p>0.05)$. However, the UR group revealed significantly more incidence of UTI at the rehabilitation unit $(\mathrm{p}<0.05)$.

In Table 3, the functional statuses of participants at transfer or admission to the rehabilitation unit are summarized. The means of MMSE, MBI, BBS, and FAC were significantly different between both groups $(\mathrm{p}<0.05)$. At the initial stage of rehabilitation, the impairments of ADL, walking ability, balance and cognitive function were worse in the patients with UR.

The functional outcomes of both groups after rehabilitation program and comparison of outcomes between the groups are revealed in Fig. 1. Both groups showed significant improvements of all functional outcomes, including motor recovery (FMA), ADL (MBI), walking ability (FAC), balance (BBS), and cognitive function (MMSE) after re- habilitation $(\mathrm{p}<0.05)$. Comparing the outcomes between the groups using a generalized linear model analysis, the significant differences were observed in MBI, FAC, and BBS $(\mathrm{p}<0.05)$. The patients without UR revealed more prominent functional recovery of ADL, walking ability and balance.

\section{DISCUSSION}

The results of this study indicate that UR is related to poor functional status at initial stage of rehabilitation, as well as poor recovery after rehabilitation. More specifi-

Table 3. Functional outcomes at transfer to rehabilitation unit

\begin{tabular}{lccc}
\hline & $\begin{array}{c}\text { UR } \\
(\mathbf{n}=\mathbf{2 5})\end{array}$ & $\begin{array}{c}\text { Non-UR } \\
(\mathbf{n}=69)\end{array}$ & p-value \\
\hline MMSE & $14.56 \pm 9.2$ & $20.52 \pm 7.9$ & $0.03^{*}$ \\
\hline MBI & $27.44 \pm 22.7$ & $46.07 \pm 22.5$ & $0.01^{*}$ \\
BBS & $9.88 \pm 12.7$ & $20.1 \pm 15.7$ & $0.04^{*}$ \\
FAC & $0.56 \pm 0.87$ & $1.35 \pm 1.2$ & $<0.01^{*}$ \\
\hline FMA & $42.28 \pm 35.7$ & $63.52 \pm 31.8$ & 0.07 \\
\hline
\end{tabular}

Values are presented as mean \pm standard deviation.

UR, urinary retention; MMSE, Mini-Mental State Examination; MBI, Modified Barthel Index; BBS, Berg Balance Scale; FAC, Functional Ambulation Category; FMA, FuglMeyer Assessment.

${ }^{*} \mathrm{p}<0.05$.

Table 2. Comparison of characteristics between UR group and transient UR group

\begin{tabular}{|lccc|}
\hline \multicolumn{1}{c}{ Variable } & UR $(\mathbf{n}=\mathbf{2 5})$ & Transient UR $(\mathbf{n}=\mathbf{1 6})$ & p-value \\
\hline Days to bladder function recovery & NA & $2.94 \pm 1.88$ & \\
\hline $\begin{array}{l}\text { Disease duration (day) } \\
\text { Stroke lesion }\end{array}$ & $12.3 \pm 5.0$ & $11.7 \pm 6.1$ & 0.69 \\
\hline Supratentorial & 20 & & 0.53 \\
\hline Infratentorial & 5 & 14 & 0.87 \\
\hline Stroke type & & & \\
\hline Ischemic & 15 & 10 & $0.01^{*}$ \\
\hline Hemorrhagic & 10 & 6 & 0.242 \\
\hline UTI at rehabilitation unit & $18(72.0)$ & $5(31.3)$ & 0.25 \\
\hline Previous UTI history & $7(28.0)$ & $2(12.5)$ & 0.07 \\
\hline Alpha blocker & $17(68.0)$ & $8(50.0)$ & $3(18.8)$ \\
\hline Cholinergics & $3(12.0)$ & & \\
\hline
\end{tabular}

Values are presented as mean \pm standard deviation or number (\%).

UR, urinary retention; UTI, urinary tract infection.

${ }^{*} \mathrm{p}<0.05$. 

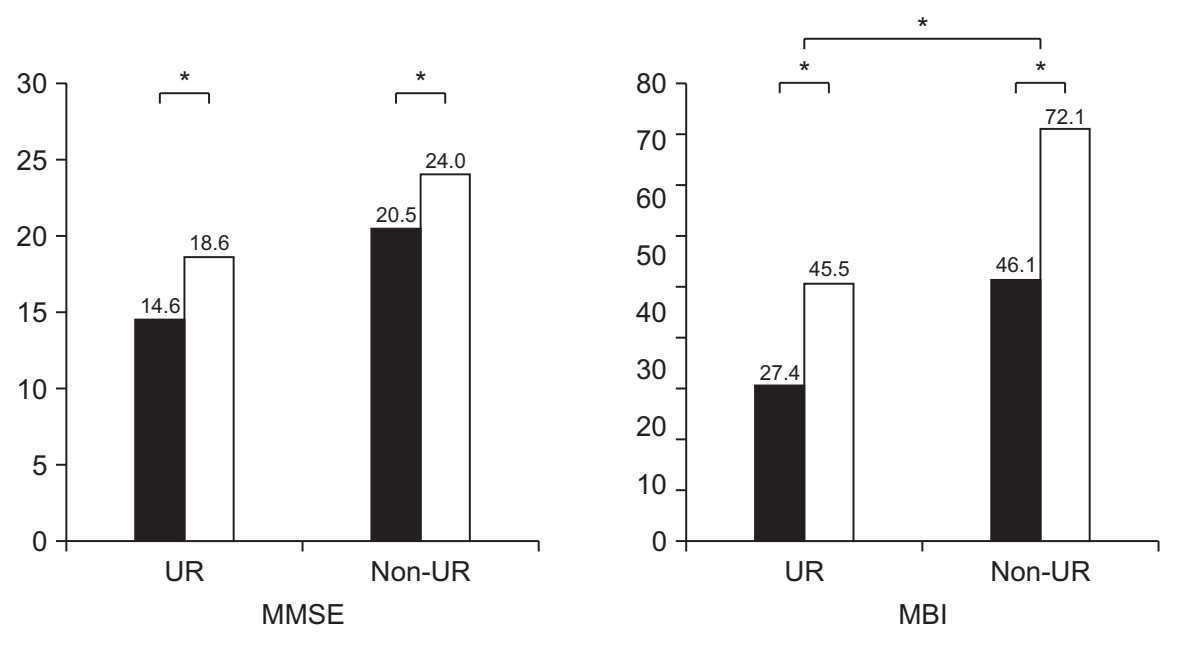

At transfer

At discharge
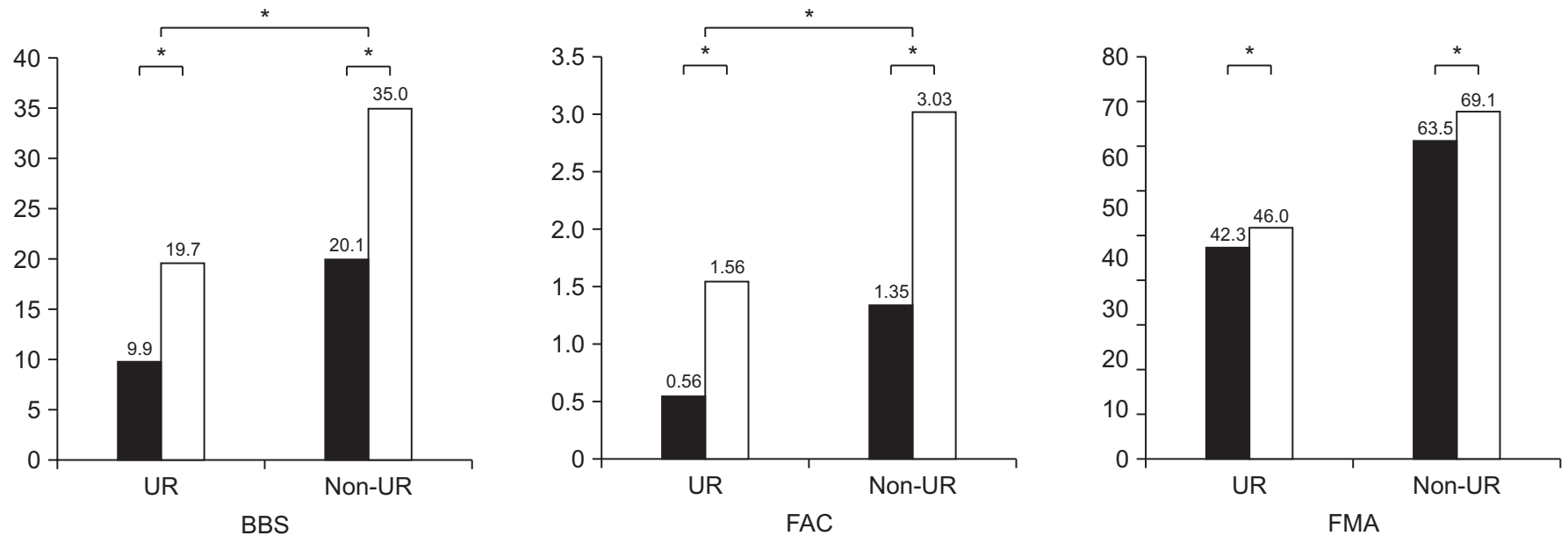

Fig. 1. Comparison of improvement in functional outcomes during rehabilitation program. UR, urinary retention; MMSE, Mini-Mental State Examination; MBI, Modified Barthel Index; BBS, Berg Balance Scale; FAC, Functional Ambulation Category; FMA, Fugl-Meyer Assessment. *p<0.05.

cally, the motor related outcomes, such as walking ability and balance, are associated with bladder dysfunction. In addition, this study further confirms that UR increases the risk of UTI.

Several studies indicate that urinary retention after stroke is strongly associated with functional status. Kong and Young [14] reported that urinary retention is strongly associated with poor functional status and explained that patients with lower functional status are likely to be bedridden; thus, voiding in bed is uncomfortable and also difficult to increase the intra-abdominal pressure to void. Hence, they reasoned that patients with lower functional status were more liable to develop UR. Wu and Baguley [15] reported that urinary retention was associated with poor clinical outcome in patients admitted for rehabilitation. Our study also indicates similar results; the pres- ence of UR was significantly associated with functional outcomes at the initial stage of rehabilitation. However, previous studies have limitations, in that PVR measurement and functional status evaluation was performed only at the early stage of rehabilitation. In this study, we measured PVR daily, from transfer to rehabilitation department up to discharge, if the PVR of patients was consistently more than $100 \mathrm{~mL}$. Furthermore, we evaluated the functional outcomes at discharge to investigate functional recovery after rehabilitation. Our results confirmed that UR is related with poor recovery after rehabilitation.

There are several risk factors for urinary retention after stroke, such as aphasia, diabetes mellitus, poor functional outcome and cognition dysfunction. A previous study reported that diabetes mellitus is strongly related with urinary retention [14]. Increased PVR volume in diabetic 
patients is caused by diabetic cystopathy, which is associated with impaired bladder sensation and decreased detrusor contractility [18]. However, our study, the presence of diabetes mellitus has no significant relevance between UR and non-UR groups. This discrepancy is probably because the severity of diabetes mellitus (diabetes duration or concomitant diabetic neuropathy) is different. One study also reports a significant association between aphasia and UR in stroke patients. Kong and Young [14] explained that the difficulty of stroke patients with aphasia to communicate their voiding problem, could induce urinary retention. In our study, 19 patients had symptoms of aphasia; however, there was no significant relation between aphasia and UR. It can be considered that influence of aphasia on UR differs according to severity or type of aphasia.

Previous reports stated that recovery of bladder function is common in stroke patients [15]. In our study, a total of $39 \%$ patients ( 16 of 41 ) had spontaneously resolved UR. Kong and Young [14] reported 22 out of 23 patients (95.6\%) showed recovery of UR; 19 patients (82.6\%) resolved by discharge, and 3 patients $(13.0 \%)$ within 2 months after discharge. In the present study, 25 patients with continuous UR did not recover the bladder function until discharge. Compared to previous studies, the relative shorter length of stay in our rehabilitation unit (20.5 \pm 7.7 days) might be related with the low recovery rate of bladder function.

Patients without UR presented greater significant functional recovery of ADL, including walking and balance. We thought that vulnerability to presence of UTI is one reason for this. Since UTI leads to the general deterioration of patients, it causes discontinuation of intensive rehabilitation program until the general condition gets resolved. Hence, management of UR after stroke is very important, and monitoring PVR and investigating risk factors for UR should be performed in subacute stage patients. Appropriate management, such as medication including alpha blockers, cholinergic, and intermittent urinary catheterization, would be helpful to avoid large PVR volume in patients identified to have UR. However, because our study was retrospective in design, we could not identify the risk factors and perform active bladder management for UR. This is a major limitation of our study.

There are several other limitations in this study. They include the small number of participants, and not considering other factors that could influence UR and UTI, such as duration of Foley catheter indwelling and lesion size. We could not evaluate PVR, functional outcomes with long-term follow-up, and did not performed urodynamic study and ultrasound of prostate to analyze the voiding dysfunction pattern and cause. Further studies are needed to address these limitations.

Although there were some limitations, the patients with UR were scanned for PVR daily, and not only at the early stage of rehabilitation. We evaluated the functional status of patients twice (at transfer, discharge), and used various scales that could assess cognition, ADL, ambulatory ability of patients. We believe these are the strengths of our study, as compared to others.

In conclusion, UR, a common complication in poststroke patients, is significantly related with poor functional status at initial stage of rehabilitation, and also with poor in subacute stage of rehabilitation. Therefore, we believe that careful monitoring of PVR by bladder scan will help to predict the functional outcome after subacute stage in stroke patients.

\section{CONFLICT OF INTEREST}

No potential conflict of interest relevant to this article was reported.

\section{ACKNOWLEDGMENTS}

This research was supported by Basic Science Research Program through the National Research Foundation of Korea (NRF) funded by the Ministry of Education (NRF2012R1A1A2043955).

\section{REFERENCES}

1. Dromerick AW, Edwards DF. Relation of postvoid residual to urinary tract infection during stroke rehabilitation. Arch Phys Med Rehabil 2003;84:1369-72.

2. Garrett VE, Scott JA, Costich J, Aubrey DL, Gross J. Bladder emptying assessment in stroke patients. Arch Phys Med Rehabil 1989;70:41-3.

3. Burney TL, Senapati M, Desai S, Choudhary ST, Badlani GH. Acute cerebrovascular accident and lower urinary tract dysfunction: a prospective correlation 
of the site of brain injury with urodynamic findings. J Urol 1996;156:1748-50.

4. Gelber DA, Good DC, Laven LJ, Verhulst SJ. Causes of urinary incontinence after acute hemispheric stroke. Stroke 1993;24:378-82.

5. Emberton M, Anson K. Acute urinary retention in men: an age old problem. BMJ 1999;318:921-5.

6. Choong S, Emberton M. Acute urinary retention. BJU Int 2000;85:186-201.

7. Yang CC, Cardenas DD. Bladder management in women with neurologic disabilities. Phys Med Rehabil Clin N Am 2001;12:91-110.

8. Dorflinger A, Monga A. Voiding dysfunction. Curr Opin Obstet Gynecol 2001;13:507-12.

9. Mustonen S, Ala-Houhala IO, Tammela TL. Longterm renal dysfunction in patients with acute urinary retention. Scand J Urol Nephrol 2001;35:44-8.

10. Curtis LA, Dolan TS, Cespedes RD. Acute urinary retention and urinary incontinence. Emerg Med Clin North Am 2001;19:591-619.

11. Kwan J, Hand P. Infection after acute stroke is associated with poor short-term outcome. Acta Neurol Scand 2007;115:331-8.
12. Kwan J, Hand P. Early neurological deterioration in acute stroke: clinical characteristics and impact on outcome. QJM 2006;99:625-33.

13. Birschel P, Ellul J, Barer D. Progressing stroke: towards an internationally agreed definition. Cerebrovasc Dis 2004;17:242-52.

14. Kong KH, Young S. Incidence and outcome of poststroke urinary retention: a prospective study. Arch Phys Med Rehabil 2000;81:1464-7.

15. Wu J, Baguley IJ. Urinary retention in a general rehabilitation unit: prevalence, clinical outcome, and the role of screening. Arch Phys Med Rehabil 2005;86: 1772-7.

16. Chen CM, Hsu HC, Tsai WS, Chang $\mathrm{CH}$, Chen KH, Hong CZ. Infections in acute older stroke inpatients undergoing rehabilitation. Am J Phys Med Rehabil 2012; 91:211-9.

17. Kim TG, Chun MH, Chang MC, Yang S. Outcomes of drug-resistant urinary retention in patients in the early stage of stroke. Ann Rehabil Med 2015;39:262-7.

18. Brown JS. Diabetic cystopathy: what does it mean? J Urol 2009;181:13-4. 